\title{
Archivos de Cardiología de México

\section{Importancia médica del glucocáliz endotelial. Parte 2: su papel en enfermedades vasculares y complicaciones de la diabetes mellitus}

\author{
Alberto C. Frati Munari* \\ Departamento de Medicina Interna, Hospital Médica Sur, México D.F., México
}

Recibido el 27 de junio de 2013; aceptado el 21 de octubre de 2013

\author{
PALABRAS CLAVE \\ Glucocáliz; \\ Disfunción endotelial; \\ Enfermedad venosa \\ crónica; \\ Aterosclerosis; \\ Sulodexida; \\ México
}

\begin{abstract}
Resumen El glucocáliz endotelial es una capa constituida por glucosaminoglicanos, proteoglicanos y glucoproteínas que cubre al endotelio en su cara luminal. La participación del deterioro del glucocáliz endotelial parece esencial en los pasos iniciales de la fisiopatología de la aterosclerosis, de las complicaciones microangiopáticas de la diabetes mellitus y de la enfermedad venosa crónica. Los factores de riesgo de la aterosclerosis como la hipercolesterolemia, la hiperglucemia, la inflamación, el exceso de sodio y las fuerzas de tensión alteradas causan deterioro del glucocáliz. Esto provoca disfunción endotelial y permite la filtración de lipoproteínas (LDL) y de leucocitos al espacio subendotelial, iniciando la formación de la placa de ateroma. En la diabetes el glucocáliz adelgazado, principalmente por estrés oxidativo, posibilita la filtración de proteínas (albuminuria) y el trastorno endotelial de la microangiopatía. La hipertensión venosa crónica altera las fuerzas de tensión y daña el glucocáliz, lo que permite la filtración de leucocitos a las partes más profundas de la pared venosa, iniciando la inflamación y el deterioro morfológico y funcional de las venas que lleva a la enfermedad venosa crónica. El tratamiento con glucosaminoglicanos (sulodexida) logra prevenir o revertir el daño al glucocáliz endotelial y algunas de sus consecuencias; es eficaz en la enfermedad venosa crónica, especialmente con úlceras venosas. También ha sido útil en aterosclerosis obliterante de miembros inferiores y en la nefropatía diabética con albuminuria.

(C) 2013 Instituto Nacional de Cardiología Ignacio Chávez. Publicado por Masson Doyma México S.A. Todos los derechos reservados.
\end{abstract}

\section{KEYWORDS}

Glycocalyx;

Endothelial

dysfunction;

Chronic venous

disease;

Atherosclerosis;

Sulodexide;

Mexico
Medical significance of endothelial glycocalyx. Part 2: Its role in vascular diseases and in diabetic complications

\begin{abstract}
Endothelial glycocalyx is a layer composed by glycosaminoglycans, proteoglycans and glycoproteins attached to the vascular endothelial luminal surface. Shredding of glycocalyx appears as an essential initial step in the pathophysiology of atherosclerosis and microangiopathic complications of diabetes mellitus, as well as in chronic venous disease. Atherosclerosis risk factors, as hypercholesterolemia (LDL), hyperglycemia, inflammation, salt excess and altered shear stress can damage glycocalyx. This lead to endothelial dysfunction and allows LDL and leukocytes to filtrate to the subendothelial space initiating atheroma plaque formation.
\end{abstract}

* Puente de Piedra 150-1-929, Toriello Guerra, Tlalpan 14050, México, D.F., México. Teléfono: +5666 5847.

Correo electrónico: AFrati@alfawassermann.com 
Degradation of glycocalyx in diabetes mellitus is mainly due to oxidative stress and enables protein filtration (albuminuria) and endothelial disorder of microangiopathy. Chronic venous hypertension brings to altered shears stress which results in shredded glycocalyx, this allows leukocytes to migrate into venous wall and initiate inflammation leading to morphologic and functional venous changes of the chronic venous disease. Treatment with glycosaminoglycans (sulodexide) prevents or recovers the damaged glycocalyx and several of its consequences. This drug improves chronic venous disease and promotes healing of chronic venous ulcers. It has also been useful in peripheral arterial obstructive disease and in diabetic nephropathy with albuminuria.

( 2013 Instituto Nacional de Cardiología Ignacio Chávez. Published by Masson Doyma México S.A. All rights reserved.

\section{Introducción}

El glucocáliz endotelial es una capa constituida por glucosaminoglicanos, proteoglicanos y glucoproteínas que forma parte del endotelio de todos los vasos sanguíneos, al que cubre en su cara luminal. A pesar de que esta tenue estructura se ha observado apenas desde hace pocas décadas, se conoce su participación en la fisiología vascular, particularmente en la del endotelio. La importancia del glucocáliz endotelial en las enfermedades que involucran los vasos sanguíneos se ve apoyada por: a) diversos factores de riesgo de enfermedades vasculares (arteriales, venosas, microvasculares) son capaces de dañar el glucocáliz; b) el deterioro del glucocáliz produce alteraciones comunes a las que ocurren en esas enfermedades vasculares; c) el tratamiento con glucosaminoglicanos específicos restablece el glucocáliz y tiene aplicaciones terapéuticas en algunos padecimientos vasculares ${ }^{1}$.

Las alteraciones del glucocáliz endotelial parecerían ser esenciales en la fisiopatología de ciertas enfermedades de las arterias (aterosclerosis), de las venas (enfermedad venosa crónica $[E V C]$ ) y de la microcirculación (complicaciones diabéticas microangiopáticas) de la siguiente manera:

\section{En la aterosclerosis}

La característica histopatológica fundamental de la aterosclerosis es el engrosamiento de la íntima con depósitos subendoteliales de LDL que se oxidan y son fagocitadas por macrófagos, formando células espumosas; la acumulación de estas células se rodea de una capa fibrosa, formando la placa ateromatosa. Esta puede estrechar la luz arterial y eventualmente se rompe, activando localmente la coagulación para formar un trombo que ocluye la luz arterial y provoca un infarto.

En la fisiopatología de la aterosclerosis es determinante la participación de la inflamación. En los focos de ateroma en la íntima existe un incremento en el número de monocitos (macrófagos) y linfocitos provenientes de la circulación y de células musculares lisas que migran de la capa media a la íntima, así como una alteración en la proporción de los distintos tipos de colágeno, de proteoglicanos, de glucosaminoglicanos y metaloproteinasas de la matriz y de sus inhibidores tisulares (MMP/TIMP) ${ }^{2}$. Un paso clave en el desarrollo de la lesión es el reclutamiento de monocitos que inicia con la unión leucocitos-células endoteliales, la liberación de quimiocinas que atraen a los monocitos al subendotelio y la maduración de los monocitos a macrófagos con la liberación de moléculas proinflamatorias por parte de los macrófagos y plaquetas activadas ${ }^{3}$.

Ciertos factores de riesgo cardiovascular, como la hipercolesterolemia, la hiperglucemia y la hipernatremia, deterioran y adelgazan el glucocáliz endotelial. No se ha investigado la influencia de la hipertensión arterial en el glucocáliz, pero probablemente actúe modificando las fuerzas de cizallamiento o de tensión. Además, tanto en la aterosclerosis que sucede naturalmente, como en la experimental, las lesiones no ocurren al azar, sino que aparecen preferentemente en los arcos, bifurcaciones y ramificaciones, lugares en los que existen turbulencias del flujo sanguíneo y alteraciones de las fuerzas de tensión ${ }^{4}$. En estos mismos lugares el glucocáliz endotelial está adelgazado en comparación con las zonas lineales de los mismos vasos ${ }^{5}$. En células endoteliales en cultivo se demostró que el flujo laminar sostenido regula a la baja los genes proaterogénicos como la proteína quimiotáctica de monocitos-1 (MCP-1) y otros que facilitan la acumulación de lípidos, mientras que regula al alza los genes que detienen el crecimiento. En cambio, el flujo alterado produce los cambios contrarios, proateroscleróticos ${ }^{6}$. La modulación de la síntesis del glucocáliz endotelial ejercida por las fuerzas de cizallamiento alteradas puede contribuir a adelgazar el glucocáliz y tornarlo vulnerable al desarrollo de aterosclerosis en las bifurcaciones ${ }^{7}$.

La hiperglucemia causa disfunción endotelial dosis dependiente, incrementa la producción de especies reactivas de oxígeno (ROS) y reduce la respuesta a las fuerzas de tensión ${ }^{8}$. La disminución de respuesta a estas fuerzas parece ser debida a que la hiperglucemia al dañar el glucocáliz afecta las propiedades mecanotransductoras que transmiten la información a las células endoteliales ${ }^{9}$. Esta alteración de la respuesta también puede explicar, al menos en parte, la mayor incidencia de enfermedad cardiovascular en los diabéticos.

Ya se vio que el deterioro del glucocáliz va seguido de disfunción endotelial, permite la filtración de moléculas de lipoproteínas a la zona subendotelial y la adhesión de 


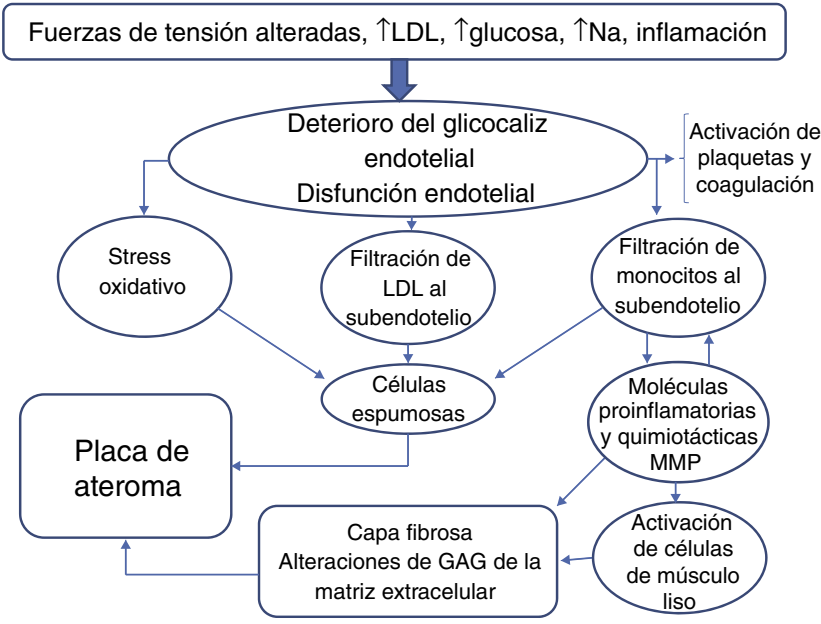

Figura 1 Fisiopatología de la aterosclerosis: esquema de la influencia de los factores de riesgo en el glucocáliz endotelial y el desarrollo ulterior de aterosclerosis.

leucocitos a las células endoteliales, pasos esenciales en la fisiopatología de la aterosclerosis. Además incrementa la adhesión de las plaquetas al endotelio y puede activar la coagulación sanguínea ${ }^{1}$.

En la figura 1 se refleja un esquema simplificado de la fisiopatología de la aterosclerosis que muestra el deterioro del glucocáliz como un paso inicial.

\section{En enfermedad venosa crónica}

Existen diversas circunstancias que originan o predisponen a la EVC y que actúan incrementando en forma prolongada la presión venosa en los miembros inferiores. En una encuesta reciente que incluyó a 11,704 pacientes, las más importantes fueron: estancia prolongada de pie (en el $70 \%$ de los casos), historia familiar de EVC (50-56\%), obesidad (51$54 \%$ ) y multiparidad (80\%); además había trombosis venosa profunda previa en el 32-40\%; había más enfermos en el grupo > 60 años de edad que en más jóvenes ${ }^{10}$.

El grado de hipertensión venosa se correlaciona con la gravedad de la EVC, debido a que causa alteraciones de las fuerza de tensión que actúan sobre el glucocáliz endotelial y lo deteriora, permitiendo la adhesión y paso de leucocitos a la pared venosa ${ }^{11,12}$. Los cambios en las fuerzas hemodinámicas en las paredes venosas, incluyendo las turbulencias y la presión, inducen inflamación y subsecuentemente remodelación de las paredes y válvulas venosas, que son los mecanismos subyacentes fundamentales en la EVC, incluyendo telangiectasias, venas reticulares y venas varicosas ${ }^{13}$. Ono et al. ${ }^{14}$ encontraron infiltración de las paredes y válvulas venosas por leucocitos (monocitos-macrófagos) en todas las muestras de individuos con EVC y en ninguna de los individuos control. Estos procesos se acompañan de alteraciones en la relación MMP/TIMP, elevación de citocinas, factores de crecimiento (TGF- $\beta_{1}$, FGF- $\beta$ ) alteraciones de la matriz extracelular, inflamación con infiltración y activación de neutrófilos ${ }^{15}$. Las alteraciones de las paredes y válvulas venosas ocurren más tempranamente en el sistema venoso superficial que en el profundo que se encuentra envuelto y apoyado por fascias y músculos.

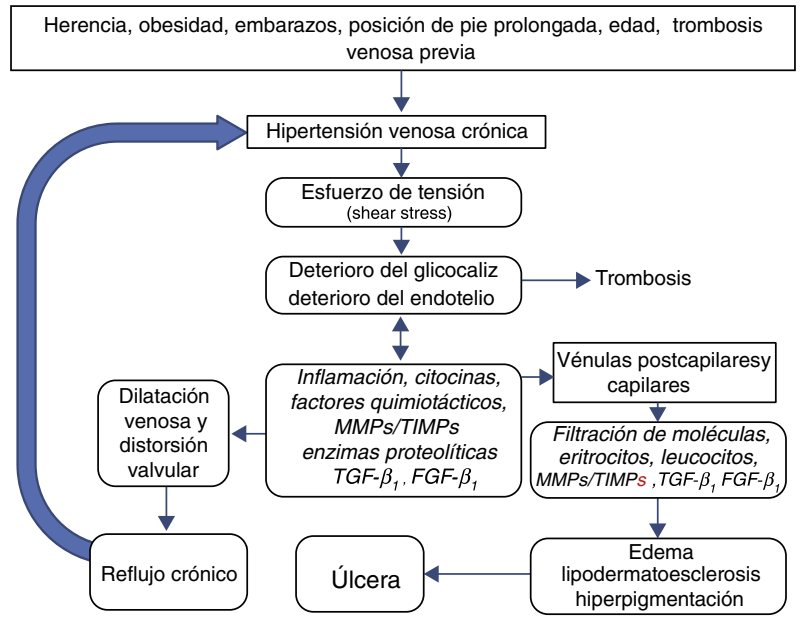

Figura 2 Fisiopatología de la enfermedad venosa crónica (EVC): se ilustra el efecto de la hipertensión venosa crónica en el deterioro del glucocáliz como paso fundamental en el desarrollo de la EVC.

Diversos estudios han mostrado que las paredes y válvulas de las venas varicosas sufren profundas alteraciones morfológicas, con engrosamiento en unos segmentos y adelgazamiento en otros, dilatación, elongación, adelgazamiento e incluso desaparición de las válvulas venosas. La incompetencia de las válvulas venosas resulta de la inflamación, dilatación o deterioro de las paredes venosas, erosiones por coágulos o anormalidades heredadas. La incompetencia valvular permite el reflujo venoso que incrementa aún más la presión y el estrés de fricción que subyace en las manifestaciones de la EVC ${ }^{16}$.

La hipertensión venosa se transmite a las vénulas y al extremo venoso de los capilares. Las alteraciones derivadas del deterioro del glucocáliz en los capilares y vénulas permiten la filtración de agua (edema), de eritrocitos (dermatitis ocre), de macromoléculas y de células inflamatorias (lipodermatoesclerosis) que pueden separar las capas de la piel y causar ulceración crónica en las piernas ${ }^{17}$. La úlcera venosa crónica es la etapa más avanzada de la EVC; ocurrió en el $12.5 \%$ de 11,704 pacientes, especialmente en > 60 años de edad $^{10}$.

Además, los senos de las válvulas venosas son sitios frecuentes del inicio de trombosis que pueden complicar la EVC, causando tromboflebitis de inicio en las várices (varicoflebitis) $^{18}$. El deterioro del glucocáliz en esos lugares promueve la activación de la coagulación.

Un esquema simplificado de la fisiopatología de la EVC (fig. 2) incluye los siguientes pasos:

1. Los factores predisponentes causan incremento prolongado en la presión venosa.

2. La hipertensión venosa crónica, por acción de las fuerzas físicas de cizallamiento (shear stress) daña el glucocáliz en primer término y después las células endoteliales.

3. El deterioro del glucocáliz permite el paso de leucocitos y moléculas proinflamatorias a la zona subendotelial.

4. En las venas la inflamación alcanza el espacio subendotelial y la capa muscular, causando alteraciones de la matriz extracelular y del músculo liso venoso. Como 
resultado la pared venosa se debilita, permitiendo que se dilate, formando las várices.

5. En las válvulas venosas la inflamación causa distorsión e incluso desaparición de las válvulas, lo cual acentúa la hipertensión venosa, empeorando el problema.

6. En la microcirculación, especialmente en las vénulas y en el extremo venoso de los capilares, el paso de leucocitos, moléculas inflamatorias y proteínas por fuera de la delgada pared vascular origina edema, oscurecimiento de la piel por extravasación de eritrocitos (dermatitis ocre), inflamación y endurecimiento de la piel (lipodermatoesclerosis) y finalmente separación de las capas de la piel y formación de úlceras crónicas de las piernas.

\section{Microangiopatía y complicaciones crónicas de la diabetes}

La microangiopatía es típicamente una consecuencia de la diabetes mellitus y se manifiesta principalmente como nefropatía, retinopatía y neuropatía. La microalbuminuria es una fase temprana de la nefropatía diabética, pero también ocurre en individuos sin diabetes mellitus.

La microalbuminuria es un importante factor de riesgo cardiovascular en la población general y es el paso inicial en la nefropatía diabética. La excreción de albúmina urinaria incluso en individuos no diabéticos guarda una relación con la aterosclerosis en arterias de mediano calibre ${ }^{19}$, se asocia con disfunción endotelial sistémica y puede observarse en diversas condiciones asociadas con daño endotelial ${ }^{20}$.

Hace más de 20 años Deckert et al. llamaron la atención sobre la albuminuria en diabéticos tipo 1 como marcador de riesgo independiente de retinopatía diabética y macroangiopatía, además de ser un indicador de enfermedad renal, y concluyeron que implicaba una disfunción endotelial generalizada que obedecía a una causa común, propusieron como alteración subyacente a trastornos de los proteoglicanos con heparán sulfato de la matriz extracelular vascular ${ }^{21}$. Con los conocimientos actuales sobre el glucocáliz endotelial, el deterioro de este emerge como un mejor candidato para explicar la disfunción endotelial y muchos de los trastornos comunes a la diabetes mellitus: microalbuminuria, microangiopatía (retinopatía, nefropatía, neuropatía) y macroangiopatía (aterosclerosis).

Después de un análisis detallado de los distintos mecanismos que pueden dañar la barrera de filtración glomerular, Satchell y Tooke $^{20}$ proponen que el glucocáliz dañado por ROS y por TNF- $\alpha$ (incrementados en la hiperglucemia) es la mejor explicación de la microalbuminuria en ausencia de daño renal observable al microscopio. Efectivamente, el deterioro del glucocáliz inducido por hiperglucemia probablemente está mediado por estrés oxidativo ya que puede impedirse con un poderoso antioxidante, la $\mathrm{N}$-acetilcisteína ${ }^{22}$. La fuente de las ROS podría ser la producción mitocondrial del radical superóxido en la misma célula endotelial como consecuencia de la hiperglucemia, en forma directa y a través de la activación de varias vías metabólicas ${ }^{23}$, o bien proveniente de los neutrófilos activados ya que la degradación enzimática del glucocáliz se acompaña de incremento en la adhesión endotelial y activación de los neutrófilos ${ }^{24-26}$. La inflamación, mediada por adipocinas y TNF- $\alpha$ en los individuos con sobrepeso, también podría contribuir al daño del glucocáliz.

Un esquema que incluye al glucocáliz en la fisiopatología de las complicaciones crónicas de la diabetes mellitus se resume en la figura 3.

\section{Implicaciones terapéuticas}

El descubrimiento del papel de las alteraciones del glucocáliz endotelial en la fisiopatología de padecimientos vasculares ha hecho suponer que esta estructura puede ser un buen objetivo terapéutico ${ }^{27-30}$. Desde luego, el primer paso es evitar o controlar las causas primarias del deterioro del glucocáliz endotelial y con ello evitar la enfermedad subsecuente. En este sentido, es interesante que el tratamiento con rosuvastatina durante 8 semanas en pacientes con hipercolesterolemia heterocigota es capaz de restaurar parcialmente el volumen sistémico del glucocáliz, que se encuentra disminuido respecto a los controles sanos ${ }^{31}$.

En experimentos animales, la adición de antioxidantes (SOD, N-acetilcisteína) previene o mejora el deterioro del glucocáliz ${ }^{32}$, pero en la clínica el único antioxidante que ha mostrado algún beneficio es el ácido $\alpha$-lipoico en la neuropatía diabética ${ }^{33}$.

Se ha investigado la administración de glucosaminoglicanos en el tratamiento, ya que son componentes del glucocáliz; el más ampliamente estudiado es la sulodexida. Esta es un glucosaminoglicano constituido por heparán sulfato y dermatán sulfato que administrado por vía parenteral $\mathrm{u}$ oral se localiza rápidamente en el endotelio vascular y tiene actividad antitrombótica, antiinflamatoria, profibrinolítica e hipolipidemiante, entre otras ${ }^{34,35}$. Se han demostrado las siguientes acciones de la sulodexida respecto al glucocáliz y al endotelio vascular: a) restaura el glucocáliz y las propiedades de barrera a la albúmina de las células endoteliales en cultivo sometidas a hiperglucemia ${ }^{36}$; b) normaliza la sintasa de NO que está disminuida en el riñón de la rata diabética y reduce la albuminuria ${ }^{37}$; c) revierte las alteraciones morfológicas de la íntima y la disfunción endotelial de las ratas diabéticas y previene el engrosamiento de la íntima inducido por estímulo mecánico ${ }^{38-40}$; d) en la isquemia-reperfusión experimental inhibe las alteraciones en la permeabilidad del glucocáliz y del estrés oxidativo ${ }^{41}$, atenúa la isquemia y reduce el depósito de marcadores de inflamación (PCR, MAC) ${ }^{42} ; e$ ) inhibe la producción de mediadores de inflamación inducidos por glucotoxicidad: ROS, proteína quimiotáctica de monocitos-1 (MCP-1) e interleucina $\left.6(\mathrm{IL}-6)^{43} ; f\right)$ en diabéticos tipo 2 restaura el espesor del glucocáliz endotelial que se encuentra disminuido y normaliza la tasa de escape transcapilar de albúmina ${ }^{44}$; g) previene el daño de los nervios periféricos en ratas diabéticas por estreptozotocina ${ }^{45}$; h) disminuye la secreción de MMP-9 por los leucocitos probablemente por interacción con los sitios de unión de las MMP al cinc, modulando así la inflamación de la pared venosa en la EVC ${ }^{46}$. La inhibición de las MMP por glucosaminoglicanos, como sulodexida, representa una novedosa intervención terapéutica para limitar la progresión de venas varicosas a EVC y ulceración de las piernas.

En la clínica, la administración de sulodexida ha sido eficaz para el tratamiento de la EVC ${ }^{47}$, especialmente en las úlceras venosas ${ }^{48-50}$. Se ha observado mejoría clínica 


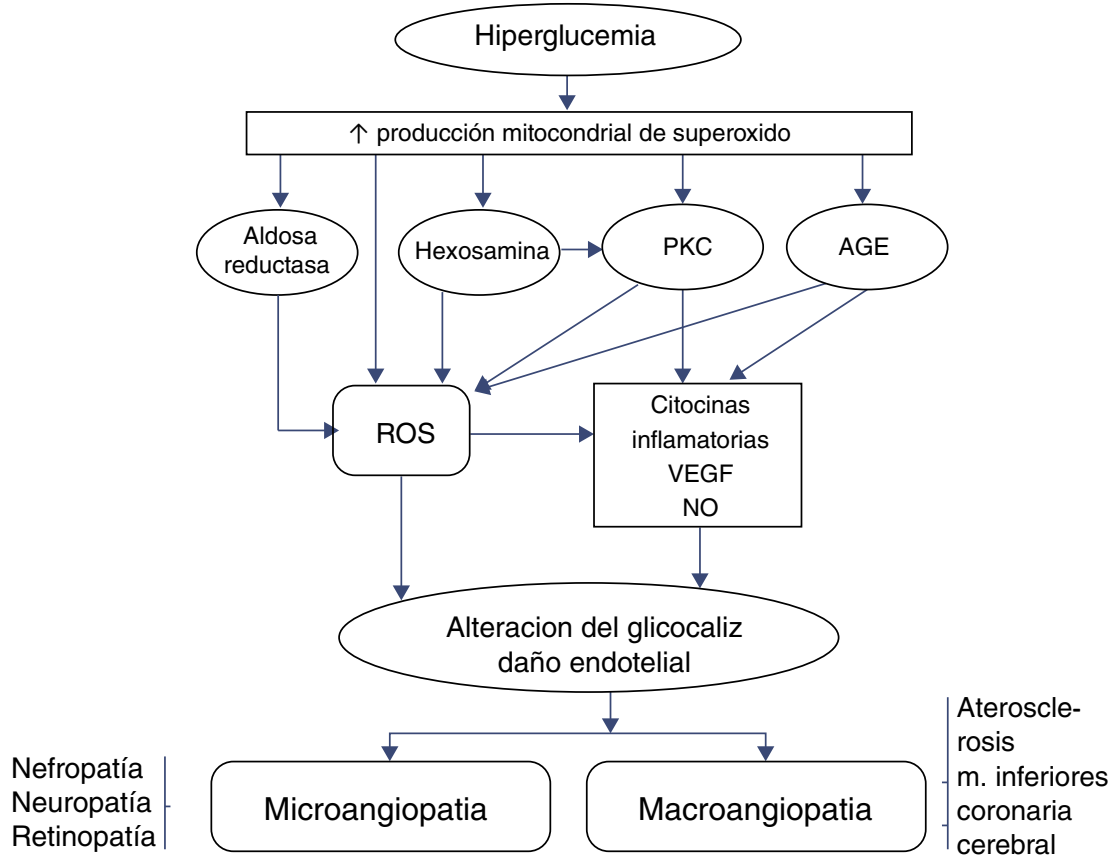

Figura 3 Fisiopatología de las complicaciones crónicas microvasculares y macrovasculares de la diabetes mellitus. La hiperglucemia a través de especies reactivas de oxígeno (ROS) dañaría el glucocáliz endotelial, lo que lleva a las complicaciones (ver texto). AGE: productos finales de glucación avanzada PKC: proteincinasa $C$.

en arteriopatía obstructiva de los miembros inferiores ${ }^{51,52}$, incluyendo macroangiopatía diabética ${ }^{53}$, y ha sido de utilidad para reducir la albuminuria en pacientes con diabetes mellitus tipo 1 y tipo $2^{54,55}$. Algunas evidencias sugieren que otro glucosaminoglicano (mesoglicano), que también contiene heparán sulfato y dermatán sulfato, puede ser útil en la insuficiencia venosa crónica ${ }^{56}$.

\section{Conclusiones}

El glucocáliz endotelial es esencial en la fisiología y la homeostasis vascular, especialmente por su papel modulador de las funciones endoteliales y en la relación entre los componentes sanguíneos y el endotelio vascular.

La degradación del glucocáliz endotelial ocurre por diversas agresiones, muchas de las cuales son comunes en los individuos con el estilo de vida de las sociedades occidentales: exceso de sal, hiperglucemia, hiperlipidemia, inflamación, estrés oxidativo, fuerzas de cizallamiento anormales. La lesión de esta estructura participa indudablemente en la fisiopatología de las enfermedades que afectan los vasos, al menos en las fases iniciales: aterosclerosis, microangiopatía diabética, EVC y probablemente hipertensión arterial.

La restauración del glucocáliz deteriorado aportando componentes del mismo (glucosaminoglicanos) permite un abordaje terapéutico diferente y novedoso que, además, podría añadirse al tratamiento establecido de cada enfermedad vascular. El lugar de estos (sulodexida) en la terapéutica está claro en la EVC, pero en las arteriales y microangiopáticas deberá definirse, teniendo en cuenta no solo los marcadores bioquímicos, sino también las variables clínicamente importantes y los niveles de riesgo ${ }^{57,58}$.
Es probable que en el futuro se desarrollen métodos de diagnóstico sencillos y prácticos, tal vez determinando biomarcadores de la degradación del glucocáliz, que permitan realizar más fácilmente investigaciones clínicas y posteriormente utilizarse en la práctica clínica cotidiana. El desarrollo de más intervenciones terapéuticas que actúen en esta estructura probablemente seguirá y añadirá otro recurso de tratamiento de las enfermedades vasculares.

\section{Financiación}

No se recibió patrocinio de ningún tipo para llevar a cabo este artículo.

\section{Conflicto de intereses}

El autor es director médico de Alfa Wassermann S.A. de CV, productor de sulodexida.

\section{Bibliografía}

1. Frati-Munari AC. Importancia médica del glucocáliz endotelial. Arch Cardiol Mex. 2013;83:303-12.

2. Raines EW. The extracellular matrix can regulate vascular cell migration, proliferation, and survival: relationship to vascular disease. Int J Exper Pathol. 2000;81:173-82.

3. Libby P, Ridker PM, Hansson GK. Inflammation in aterosclerosis: From pathophysiology to practice. J Am Coll Cardiol. 2009;54:2129-38.

4. Chiu JJ, Chen S. Effects of disturbed flow on vascular endothelium: Pathophysiological basis and clinical perspectives. Physiol Rev. 2011;91:327-87.

5. Van den Bergh BM, Spaan JAE, Rolf TM, et al. Atherogenic región and diet diminish glycocalyx dimensión and increase intima 
media ratios at the murine carotid artery bifurcation. Am J Physiol Heart Circ Physiol. 2006;290:H915-20.

6. Chien SS. Molecular and mechanical bases of focal lipid accumulation in arterial wall. Prog Biophys Mol Biol. 2003;83:131-51.

7. Governeur M, van den Berg B, Nieuwdorp M, et al. Vasculoprotective properties of the endotelial glycocalyx: Effects of fluid shear stress. J Intern Med. 2006;259:393-400.

8. Gross ER, LaDisa JR, Weihrauch D, et al. Reactive oxygen species modulate coronary wall shear stress and endothelial function during hyperglycemia. Am J Physiol Heart Circ Physiol. 2003;284:H1552-9.

9. Kelly R, Ruane-O'Hora T, Noble MIM, et al. Differential inhibition by hyperglycaemia of shear stress-but not acetylcholinemediated dilatation in the artery of anaesthetized pig. J Physiol. 2006;573:133-45.

10. Agus GB, Mattana P, Carelli F. Monitor 2010: Italian epidemiological survey on chronic venous diseases. Acta Phlebol. 2012;13:9-17.

11. Secomb TW, Hsu R, Pries AR. Effect of the endotelial surface layer on transmission of fluid shear stress to endothelial cells. Biorrheology. 2001;38:143-50.

12. Becker BF, Chappel D, Bruegger D, et al. Therapeutic strategies targeting the endothelial glycocalyx: Acute deficits, but great potential. Cardiovasc Res. 2010;87:300-10.

13. Bergan JJ, Schmid-Schönbein GW, Coleridge-Smith PD, et al. Chronic venous disease. N Engl J Med. 2006;355:488-98.

14. Ono T, Bergan JJ, Schmid-Schönbein GW, et al. Monocyte infiltration into venous valves. J Vasc Surg. 1998;27:158-66.

15. Nicolaides AN. Chronic venous disease and the leukocyteendothelium interaction: From symptoms to ulceration. Angiology. 2005;56 Suppl 1:S11-9.

16. Raffetto JD, Khalil RA. Mechanisms of varicose vein formation: Valve dysfunction and wall dilation. Phlebology. 2008;23: 85-98.

17. Simka M. Cellular and molecular mechanisms of venous ulcer development. The «puzzle» theory. Int Angiol. 2010;29:1-19.

18. Brooks EG, Trotman W, Wadsworth MP, et al. Valves of the deep venous system: an overlooked risk factor. Blood. 2009;114:1276-9.

19. Furtner M, Kielchl S, Mair A, et al. Urinary albumin excretion is independently associated with carotid and femoral artery aterosclerosis in the general population. Eur J Cardiol. 2005;26:279-87.

20. Satchell SC, Tooke JE. What is the mechanism of microalbuminuria in diabetes: A role for the glomerular endothelium? Diabetologia. 2008;51:714-25.

21. Deckert T, Feldt-Rasmussen B, Borch-Johnsen K, et al. Albuminuria reflects widespread vascular damage. The Steno hypothesis. Diabetologia. 1989;32:219-26.

22. Nieuwdorp M, van Haeften TW, Gouverneur MCLG, et al. Loss of endotelial glycocalyx during acute hyperglycemia coincides with endothelial dysfunction and coagulation activation in vivo. Diabetes. 2006;55:480-6.

23. Brownlee M. The pathobiology of diabetic complications. A unifying mechanism. Diabetes. 2005;54:1615-25.

24. Mulivor AW, Lipowsky HH. Role of glycocalyx in leukocyteendothelial cell adhesión. Am J Physiol Heart Circ Physiol. 2002;283:H1282-91.

25. Constantinescu AA, Vink H, Spaan JAE. Endothelial cell glycocalyx modulates immobilization of leukocytes at the endotelial surface. Arterioscler Thromb Vasc Biol. 2003;23:1541-7.

26. Butler LM, Rainger GE, Nash GB. A role for the endotelial glycosaminoglycan hyaluronan in neutrophil recruitment by endotelial cells cultured for prolonged periods. Exp Cell Res. 2009;315:3433-41.

27. Nieuwdorp M, Meuwese MC, Vink H, et al. The endothelial glycocalyx: A potential barrier between health and vascular disease. Curr Opin Lipidol. 2005;16:507-11.
28. Becker BF, Chappell D, Bruegger D, et al. Therapeutic strategies targeting the endothelial glycocalyx: Acute deficits, but great potential. Cardiovasc Res. 2010;87:300-10.

29. Noble MIM, Drake-Holland AJ, Vink H. Hypothesis: Arterial glycocalyx dysfunction is the first step in the atherothrombotic process. QJM. 2008;101:513-8.

30. Noble MIM, Drake-Holland AJ. Review: Hyperglycemia and the vascular glycocalyx: the key to microalbuminuria and cardiovascular diseae in diabetes mellitus? Br Med J Diab Vasc Dis. 2010;10:66-70.

31. Meuwese MC, Mooij HL, Nieuwdorp M, et al. Partial recovery of the endothelial glycocalyx upon rosuvastatin therapy in patients with heterozygous familial hypercholesterolemia. J Lipid Res. 2009;50:148-53.

32. Moseley R, Waddington RJ, Embery G. Degradation of glycosaminoglycans by reactive oxigen species derived from stimulated polymorfonucleal leukocytes. Biochem Biophys Acta. 1997; 1362:221-31.

33. Ziegler D, Ametov A, Barinov A, et al. Oral treatment with $\alpha$-lipoic acid improves symptomatic diabetic polyneuropathy. Diabetes Care. 2006;29:2365-70.

34. Haremberg J. Review of pharmacodynamics, pharmacokinetics, and therapeutic properties of sulodexide. Med Res Rev. 1998; 18:1-20.

35. Ofosu FA. Pharmacological actions of sulodexide. Sem Thromb Hemost. 1998;24:127-38.

36. Broekhuisen LN, Meuwese MC, Mooij HL, et al. Sulfated glycosaminoglycans restore glycocalyx barrier of cultured endothelial cells under hyperglycemic conditions. En: Meuwese MC, editor. Targeting the vessel wall in cardiovascular prevention. Amsterdam: Univ. Amsterdam; 2008., ISBN 9789085594024 p. 46-54 [consultado 30 Ago 2010]. Disponible en: http://dare.uva.nl/ document/112476

37. Mathison Y, Garrido MR, Israel A, et al. Efecto del glicosaminoglicano sulodexida sobre la actividad de la sintasa de óxido nítrico en la corteza renal de ratas con diabetes tipo 1. Rev Latinoamer Hipert. 2008;3:182-9.

38. Kristová V, Lisková S, Sotniková R, et al. Sulodexide improves endotelial dysfunction in streptozotocin-induced diabetes in rats. Physiol Res. 2008; 57:491-4.

39. Vasquez J, Mathison Y, Romero-Vecchione E, et al. Efecto del sulodexide sobre la capacidad de relajación y alteraciones estructurales de la arteria aorta en ratas diabéticas por estreptozotocina. Invest Clin. 2010;51:467-77.

40. Park HY, Kang S, Kim GY, et al. Inhibition of neointimal proliferation of rat carotid artery by sulodexide. J Korean Med Sci. $1997 ; 12: 210-4$

41. Rubio-Gayosso I, Platts SH, Duling BR. Reactive oxygen species mediate modification of glycocalyx during ischemia-reperfusion injury. Am J Physiol Heart Circ Physiol. 2006;290:H2247-56.

42. Lauver DA, Booth EA, White AJ, et al. Sulodexide attenuates myocardial ischemia/reperfusion injury and the deposition of C-reactive protein in areas of infarction without affecting hemostasis. J Pharmacol Exp Ther. 2005;312:794-800.

43. Ciszewicz M, Polubinska A, Antoniewicz A, et al. Sulodexide suppresses inflammation in human endothelial cells and prevents glucose cytotoxicity. Transl Res. 2009;153:118-23.

44. Broekhuizen LN, Lemkes BA, Mooij HL, et al. Effect of sulodexide on endothelial glycocalyx and vascular permeability in patients with type 2 diabetes mellitus. Diabetologia. 2010;53:2646-55.

45. Jin HY, Lee KA, Song SK, et al. Sulodexide prevents peripheral nerve damage in streptozotocin induced diabetic rats. Eur J Pharmacol. 2012;674:217-26.

46. Mannello F, Raffetto JD. Matrix metalloproteinase activity and glycosaminoglycans in chronic venous disease: The linkage among cell biology, pathology and translational research. Am J Transl Res. 2011;3:149-58. 
47. Andreozzi GM. Sulodexide in the treatment of cronic, venous disease. Am J Cardiovasc Drugs. 2012;12:73-81, http://dx. doi. org/10.2165/11599360.

48. Scondotto G, Aloisi D, Ferrari P, et al. Treatment of venous leg ulcers with sulodexide. Angiology. 1999;50:883-9.

49. Coccheri S, Scondotto G, Agnelli G, et al., Venous arm of the SUAVIS (Sulodexide Arterial Venous Italian Study) group. Randomised, double-blind, multicentre, placebo controlled study of sulodexide in the treatment of venous leg ulcers. Thromb Haemost. 2002;87:947-52.

50. Kucharzewski M, Franec A, Hoziolek $H$. Treatment of venous leg ulcers with sulodexide. Phlebologie. 2003;32: 115-20.

51. Gaddi A, Galetti C, Illuminati B, et al. Meta-analysis of some results of clinical trial son sulodexide therapy in peripheral occlusive arterial disease. J Int Med Res. 1996;24: 389-406.

52. Coccheri S, Scondotto G, Agnelli G, et al., Arterial arm of the Suavis (Sulodexide Arterial Venous Italian Study) group. Sulodexide in the treatment of intermittent claudication. Results of a randomized, double-blind, multicentre, placebo-controlled study. Eur Heart J. 2002;23:1057-65.
53. Della Marchina M, Bellucci M, Palazzini E. Medium term oral sulodexide treatment of diabetic patients suffering from peripheral arterial obstructive disease: A double-blind, placebo-controlled study. Progress Rep. 1992;4:5-15.

54. Gambaro G, Kinalska I, Oksa A, et al. Oral sulodexide reduces albuminuria in microalbuminuric and macroalbuminuric type 1 and type 2 diabetic patients: the Di.N.A.S. randomized trial. J Assoc Nephrol. 2002;13:1615-25.

55. Weiss R, Niecestro R, Raz I. The role of sulodexide in the treatment of diabetic nephropathy. Drugs. 2007;67:2681-96.

56. Tufano A, Arturo C, Cimino E, et al. Mesoglycan: Clinical evidences for use in vascular disease. In J Vasc Med. 2010;2010:396043, http: //dx.doi.org/10.1155/2010/396043 [consultado 13 Ago 2012].

57. Trejo-Gutiérrez JF, López-Jiménez F. Cardiología «basada en evidencia»: aplicaciones prácticas de la epidemiología. I. La valoración de medicamentos o terapias novedosas. Arch Cardiol Mex. 2011;81:47-52.

58. Rodríguez-Escudero JP, López-Jiménez F, Trejo-Gutiérez JF. Cardiología «basada en evidencia»: aplicaciones prácticas de la epidemiología. IV. Modelos de predicción de riesgo cardiovascular. Arch Cardiol Mex. 2012;82:66-72. 